\title{
Double Trouble in a Tropical Paradise
}

\author{
Adelyn Henry, Ganesh Kasinathan \\ Department of Internal Medicine, Segamat Hospital, Segamat, Malaysia \\ Email: ganeshkasinathan11@hotmail.com
}

How to cite this paper: Henry, A. a Kasinathan, G. (2018) Double Trouble in a Tropical Paradise. Open Access Library Journal, 5: e4325.

https://doi.org/10.4236/oalib.1104325

Received: January 11, 2018

Accepted: January 26, 2018

Published: January 29, 2018

Copyright $\odot 2018$ by authors and Open Access Library Inc.

This work is licensed under the Creative Commons Attribution International License (CC BY 4.0).

http://creativecommons.org/licenses/by/4.0/

\begin{abstract}
Introduction: Leptospirosis and typhoid fever are water borne diseases. These diseases present as acute undifferentiated fever and often can be challenging to clinicians in the developing world due to limited resources. Case Presentation: A 15-year-old Malay girl presented a week after swimming in a waterfall with fever, vomiting and lethargy. Her condition deteriorated into septic shock and respiratory distress requiring mechanical ventilation. Her blood, urine and stool cultures grew pure isolates of Salmonella typhi. The leptospira serology was positive and the leptospira microscopic agglutination test revealed a high titre of 1:400. She was diagnosed with dual infections of leptospirosis and typhoid fever. With third generation cephalosporin antibiotics, she improved. There was clearance of her blood, urine and stool cultures. Her leptospira microscopic agglutination test demonstrated a fourfold reduction in titre. Conclusion: Not only do these illnesses prove challenging to physicians to diagnose and treat due to their similarity in initial presentation, they are also life threatening to the unknowing tourist or locals who went for an innocent dip in a picturesque waterfall.
\end{abstract}

\section{Subject Areas}

Internal Medicine

\section{Keywords}

Typhoid Fever, Leptospirosis, Antibiotics, Waterfall, Septic Shock

\section{Learning Points for Clinicians}

In the tropics, an acute undifferentiated fever is common with leptospirosis, dengue fever, malaria and typhoid fever. Being endemic, they can present similarly making them indistinguishable and posing major challenges to doctors who have limited diagnostic tests and resources to make a rapid and correct diagnosis. 


\section{Introduction}

Acute febrile illness is a common entity in the developing world. Dual infections tend to have atypical manifestations which may contribute to delay in diagnosis. Various complications often arise from co-infections. Typhoid fever and leptospirosis are major public health concerns during floods in Malaysia. Typhoid fever is also an important leading cause of mortality and morbidity worldwide [1]. Both diseases are notifiable in Malaysia.

\section{Case Presentation}

A 15-year-old Malay girl with no medical history presented with fever and vomiting for a week. She complained of dyspnoea, abdominal discomfort and myalgia. Her symptoms began a week following a swim at a nearby waterfall. On examination, she was febrile at $40^{\circ} \mathrm{C}$. Her blood pressure was $100 / 64 \mathrm{mmHg}$ with a rapid bounding pulse at 112 beats per minute. She was tachypnoeic at 24 breaths per minute. Respiratory examination revealed bronchial breathing and crackles over bilateral lower lobes. There was tenderness on the right hypochondrium. Her liver and spleen were not palpable. Other systems were unremarkable. The blood parameters are shown in Table 1. Blood, stool and urine cultures were taken immediately before commencement of broad spectrum antibiotics which grew pure isolates of Salmonella typhi. Dengue serological studies, viral screening and blood films for malarial parasites were all non-reactive. Leptospira serology was reactive with the Leptospira microscopic agglutination test significant at a titre of 1:400 which reduced four-fold after 4 weeks. The ultrasound of the abdomen was normal.

She was treated with intravenous Ceftriaxone 2 grams daily. Through 10 hours of admission, her condition deteriorated necessitating intubation for respiratory distress. Chest radiographs demonstrated bilobar pneumonia. She went into septic shock complicated with disseminated intravascular coagulopathy and multiorgan dysfunctional syndrome. The ceftriaxone was adjusted to $60 \mathrm{mg} / \mathrm{kg}$ daily at a dose of 3 grams daily (adopted from the National Antibiotic Guidelines 2015, Malaysia). The hypotension was supported with a three-day course of intravenous dexamethasone and parenteral noradrenaline. The disseminated intravascular coagulopathy was treated with two cycles of cryoprecipitate, fresh

Table 1. Trend of blood parameters during the ward stay.

\begin{tabular}{|c|c|c|c|c|}
\hline $\begin{array}{l}\text { Variables (normal range } \\
\text { in standard unit) }\end{array}$ & $16 / 10 / 17$ & $17 / 10 / 17$ & $21 / 10 / 17$ & $26 / 10 / 17$ \\
\hline Total White Cell Count $\left(4-11 \times 10^{9} / \mathrm{L}\right)$ & 4.0 & 11.1 & 7.0 & 6.8 \\
\hline Platelet $\left(150-400 \times 10^{9} / \mathrm{L}\right)$ & 14 & 17 & 19 & 134 \\
\hline Aspartate transaminase $(<40 \mathrm{U} / \mathrm{L})$ & 192 & 470 & 328 & 40 \\
\hline Alanine transaminase $(<40 \mathrm{U} / \mathrm{L})$ & 42.5 & 72 & 158 & 34 \\
\hline INR (international normalised ratio) & 1.86 & 1.28 & 1.47 & 1.10 \\
\hline Creatinine (40 - 80 umol/L) & 206 & 264 & 202 & 93 \\
\hline
\end{tabular}


frozen plasma and platelet concentrates. Her multiorgan dysfunction and coagulopathy improved resulting in extubation after three days. She then completed 14 days of intravenous ceftriaxone in the isolation ward. Her stool samples were negative for Salmonella three days prior to her discharge. Other family members were also screened for asymptomatic carriage.

\section{Discussion}

Commonly, acute febrile illness is caused by a single etiological agent. Recently it has become evident that dual infections can occur either due to host related factors or vector adaptability as in the case of chikungunya and dengue or agent related factors as in the association of typhoid bacteraemia with polio virus [2]. Water borne illnesses such as leptospirosis, typhoid fever, hepatitis A and cholera thrive in rainy seasons which occur periodically in Malaysia. The risk of contracting typhoid fever and leptospirosis during flooding is generally low if there is no contamination of drinking water supplies. Frequently, outbreaks of leptospirosis and typhoid fever occur when there is contact with polluted waters from nearby rivers or massive displacement of human populations. Leptospirosis is easily transmitted via skin or mucous membrane contact with contaminated water supplies or soil contaminated with urine of rodents. On the other hand, typhoid fever is a global health problem in the developing nations and without effective treatment, it has a case fatality rate of $10 \%-30 \%$ [3].

Therefore, history taking should include a recent travel to the tropics or contaminated water sport facilities and with high clinical suspicion of leptospirosis and typhoid fever, empirical therapy needs to be started early.

In this case we have strong reasons to support the diagnosis of dual infections. Leptospira microscopic agglutination test is the gold standard for diagnosing leptospirosis. The presence of risk factors, reduction of titre from 1:400 to 1:100 after 4 weeks and a positive Leptospira serology supports the diagnosis of leptospirosis. A positive blood culture is usually diagnostic for typhoid fever. It is positive in $40 \%-60 \%$ of cases, usually early in the course of the disease [4]. Culture of the bone marrow is more sensitive, the result being positive in $80 \%$ $95 \%$ of patients with typhoid, even in patients who have been taking antibiotics for several days, regardless of the duration of illness [5].

\section{Conclusion}

Mixed infections with several pathogens such as typhoid fever and leptospirosis are common in areas of high endemicity. Careful history taking, thorough physical examination, and appropriate interpretation of laboratory results will often lead to early diagnosis. It is paramount that health authorities in such regions to educate and inform travellers and locals alike of such risks which lurk in "paradise".

\section{Consent for Publication}

Consent obtained from the patient's parents. 


\section{References}

[1] Nagshetty, K., Channappa, S.T. and Gaddad, S.M. (2010) Antimicrobial Susceptibility of Salmonella Typhi in India. Journal of Infection in Developing Countries, 4, 70-73.

[2] Mandell, G.L., Bennett, J.E., Dolin, R., et al. (2010) Mandell, Douglas, and Bennett's Principles and Practice of Infectious Diseases. 7th Edition, Churchill Livingstone, Philadelphia.

[3] Mary Sushi, K., Sivasangeeth, K., Suresh Kumar, A., Pandi, S., et al. (2014) Seroprevalence of Leptospirosis, Enteric Fever and Dengue in Acute Febrile Illness in Tamil Nadu, India. Indian Journal of Basic and Applied Medical Research, 3, 615-623.

[4] Bhutta, Z.A. (2006) Current Concepts in the Diagnosis and Treatment of Typhoid Fever. British Medical Journal, 333, 78-82. https://doi.org/10.1136/bmj.333.7558.78

[5] Parry, C.M., Hien, T.T., Dougan, G., White, N.J. and Farrar, J.J. (2002) Typhoid Fever. The New England Journal of Medicine, 347, 1770-1782. https://doi.org/10.1056/NEJMra020201 